\title{
The Process of Setting Up an Electron Microscopy Laboratory for Clinical Services.
}

\author{
Becky Radabaugh, HT (ASCP) ${ }^{\mathrm{CM}} \mathrm{QIHC}^{1}$ and Judy King, M.D., Ph.D. ${ }^{{ }^{*}}$ \\ ${ }^{1}$ West Virginia University, Department of Pathology, Morgantown, WV \\ *Currently at LSU Health, Department of Pathology \& Translational Pathobiology, Shreveport, LA
}

It all begins with an ambitious idea, a gut feeling, really. A feeling that electron microscopy might be worth the investment, an investment of time, money and additional resources. Do we have the right resources to make this happen? Can we make this successful? Will the benefits outweigh the drawbacks? Do we have the staff to make this possible? These questions and many more like them are important thoughts often associated with that feeling. How those questions get answered will determine whether an idea grows into fruition or stays just an idea.

Most electron microscopy laboratories were established when the technology was new and considered state of the art for that time period. With more technologies available, like immunohistochemistry and fluorescent capabilities, setting up new EM laboratories is not common practice these days. Some people believe that electron microscopy is a dying art, that it has seen its best days. However, transmission electron microscopy (TEM) is an integral part of the evaluation of some clinical pathologic samples, such as medical renal biopsies, transplant renal biopsies, and cilia. For these reasons and several others, TEM will never be a dying art, but simply just underutilized and underappreciated.

At West Virginia University (WVU), the idea of starting up an electron microscopy service came from the Chair of the Pathology Department in 2011, who then presented the idea to the departmental administrator. What is unique about WVU is that the major piece of equipment, the costliest piece of equipment, was quietly sitting in a locked room in the basement of the building, a JEOL JEM 1010 Transmission Electron Microscope with plate film, which was purchased in 1995. Although WVU had an electron microscopy service at one point, it was closed in early 2003 and most equipment and laboratory space was reallocated to different departments and services. In 2011, a technician was recruited to begin the daunting task of setting up a clinical electron microscopy laboratory from "scratch," starting with only an empty laboratory space and no prior EM experience. In 2012, a renal pathologist with years of electron microscopy experience was brought on board as Medical Director of the newly established electron microscopy laboratory (Figure 1).

With clinical renal biopsy interpretation, speed is of essence, so specialized equipment was purchased to optimize turn-around time (Figure 2). The lab purchased a Milestone KOS Microwave HistoStation to rapidly process clinical samples (Figure 2a). Manual processing time for a kidney biopsy was approximately $9 \frac{1}{2}$ hours of processing and 12-15 hours of polymerization in a $70^{\circ} \mathrm{C}$ oven. The KOS processor provides a sample ready to be sectioned within 5 hours of receipt. We also purchased a Leica EM UC7 Ultra-Microtome and utilize Diatome diamond knives for our sectioning (Figure 2c). Perhaps the most important purchase of all was a digital imaging system to replace the plate film of the TEM (Figure 2b). This system consists of an AMT Hamamatsu ORCA-HR Digital Camera (Figure 2b) and software which has greatly improved the imagery in all aspects (quality, ease, etc.) (Figure 3).

The Electron Microscopy Laboratory at WVU has seen a steady percent (\%) increase in its first three full years of operation (Figure 4). For us, it certainly wasn't easy and there were plenty of bumps in the road but that feeling, that one idea, turned out to be a risk worth taking. 

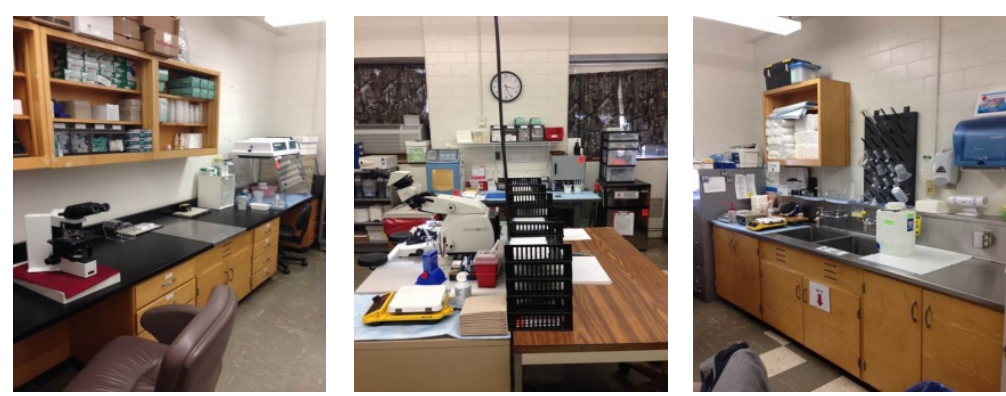

Figure 1: Electron Microscopy Laboratory at West Virginia University (WVU).
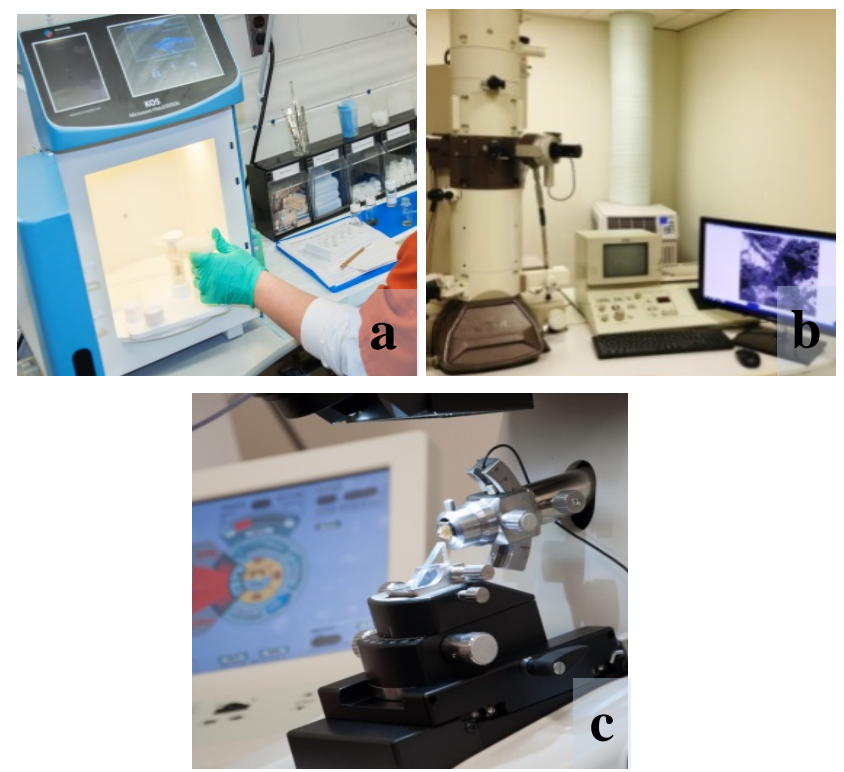

Figure 2: Instrumentation Utilized for EM Services at WVU

a: KOS Microwave Processor b: JEOL JEM 1010 Electron Microscope with AMT Hamamatsu ORCA-HR Digital Camera c: Leica EM UC7 Ultra-Microtome
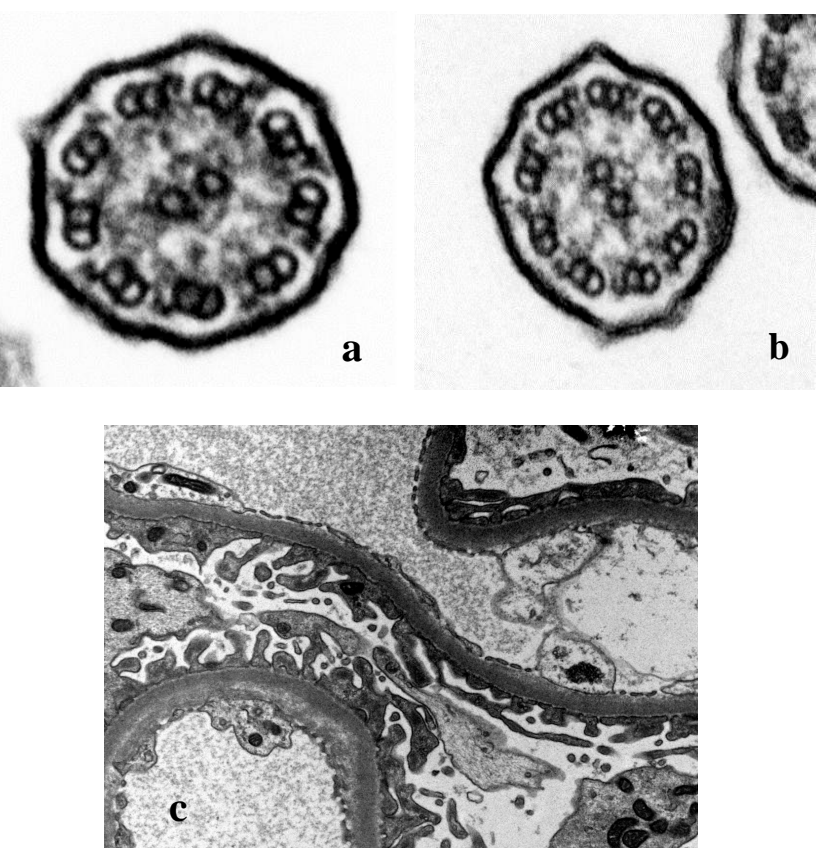

Figure 3: Digital Photographs of Clinical Samples.

a and b: Normal Cilia

c: Glomerular Basement Membranes

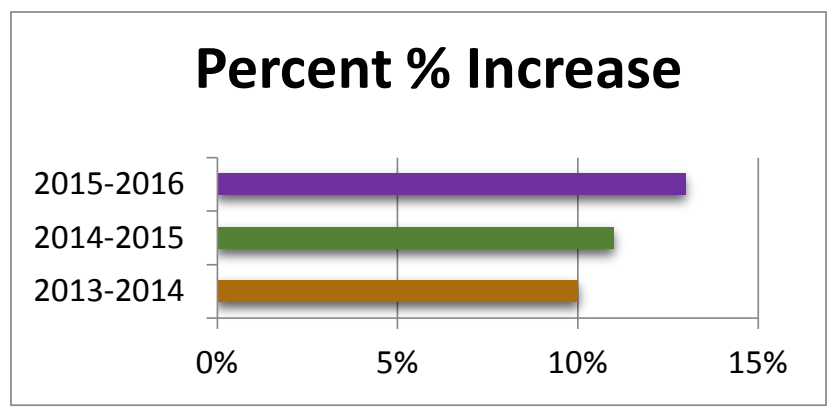

Figure 4: Percent Increase of Clinical Samples 